\title{
CCN2/CTGF is required for matrix organization and to protect growth plate chondrocytes from cellular stress
}

\author{
Faith Hall-Glenn • Armen Aivazi • Lusi Akopyan • \\ Jessica R. Ong • Ruth R. Baxter • Paul D. Benya • \\ Roel Goldschmeding • Frans A. van Nieuwenhoven • \\ Ernst B. Hunziker • Karen M. Lyons
}

Received: 8 January 2013 / Accepted: 22 April 2013 / Published online: 13 May 2013

(C) The Author(s) 2013. This article is published with open access at Springerlink.com

\begin{abstract}
CCN2 (connective tissue growth factor (CTGF/CCN2)) is a matricellular protein that utilizes integrins to regulate cell proliferation, migration and survival. The loss of CCN2 leads to perinatal lethality resulting from a severe chondrodysplasia. Upon closer inspection of $\mathrm{Ccn} 2$ mutant mice, we observed defects in extracellular matrix (ECM) organization and hypothesized that the severe chondrodysplasia caused by loss of CCN2 might be associated with defective chondrocyte survival. Ccn2 mutant growth plate chondrocytes exhibited enlarged endoplasmic reticula (ER), suggesting cellular stress. Immunofluorescence analysis confirmed elevated stress in Ccn2 mutants, with reduced stress observed in $C c n 2$ overexpressing transgenic mice. In vitro studies revealed that $C c n 2$ is a stress responsive gene in
\end{abstract}

Electronic supplementary material The online version of this article (doi:10.1007/s12079-013-0201-y) contains supplementary material, which is available to authorized users.

F. Hall-Glenn · A. Aivazi $\cdot$ L. Akopyan · J. R. Ong · K. M. Lyons Department of Molecular, Cell and Developmental Biology, University of California, Los Angeles, Los Angeles,

CA 90095, USA

R. R. Baxter $\cdot$ P. D. Benya $\cdot$ K. M. Lyons

Department of Orthopaedic Surgery, University of California,

Los Angeles, Los Angeles, CA 90095, USA

R. Goldschmeding

Department of Pathology, University of Utrecht, Utrecht, Netherlands

F. A. van Nieuwenhoven

Department of Physiology, Maastricht University, Maastricht,

Netherlands

E. B. Hunziker

Centre of Regenerative Medicine for Skeletal Tissues, Department

of Clinical Research, University of Bern, Bern, Switzerland chondrocytes. The elevated stress observed in Ccn2-/chondrocytes is direct and mediated in part through integrin $\alpha 5$. The expression of the survival marker NFKB and components of the autophagy pathway were decreased in $C \mathrm{Cn} 2$ mutant growth plates, suggesting that $\mathrm{CCN} 2$ may be involved in mediating chondrocyte survival. These data demonstrate that absence of a matricellular protein can result in increased cellular stress and highlight a novel protective role for CCN2 in chondrocyte survival. The severe chondrodysplasia caused by the loss of CCN2 may be due to increased chondrocyte stress and defective activation of autophagy pathways, leading to decreased cellular survival. These effects may be mediated through nuclear factor $\mathrm{KB}(\mathrm{NF} \kappa \mathrm{B})$ as part of a $\mathrm{CCN} 2 /$ integrin/NFKB signaling cascade.

\author{
K. M. Lyons $(\bowtie)$ \\ Department of Orthopaedic Surgery, Orthopaedic Hospital \\ Research Center, University of California Los Angeles, \\ 615 Charles E Young Drive South 501, \\ Los Angeles, CA 90095, USA \\ e-mail: klyons@mednet.ucla.edu
}

Present Address:

A. Aivazi

School of Medicine, Stony Brook University, East Loop Road,

Stony Brook, NY, USA

Present Address:

L. Akopyan

Western University of Health Sciences College of Pharmacy,

309 E. Second street,

Pomona, CA, USA 
Keywords Cartilage $\cdot \mathrm{CCN} 2 / \mathrm{CTGF} \cdot$ Chondrocytes Cellular stress $\cdot$ Nuclear factor $\mathrm{kB} \cdot$ Autophagy

\section{Introduction}

Chondrocytes secrete large amounts of extracellular matrix (ECM) as they differentiate. Disruptions in this process often result in chondrodysplasias, a diverse group of congenital birth defects characterized by short stature and skeletal deformity. The most common causes of chondrodysplasias are mutations that lead to structural alterations in genes that encode ECM proteins. These alterations prevent proper protein folding and maturation, leading to their retention in the endoplasmic reticulum (ER), and/or the incorporation of the mutated product into the ECM, interfering with matrix structure and function (Bateman et al. 2009).

ER enlargement is a hallmark of defective protein folding and cellular stress. ER and other forms of cellular stress activate the Unfolded Protein Response (UPR), an adaptive mechanism to restore cell homeostasis and viability (Rasheva and Domingos 2009). The UPR ameliorates cellular stress by attenuating protein synthesis and increasing chaperone protein levels to facilitate correct protein folding and clearing of misfolded protein aggregates. The glucose-regulated protein 78 (GRP78) also known as BiP, is a resident ER chaperone and the main initiator of the UPR (Bertolotti et al. 2000). BiP activates UPR effector molecules that function to restore cellular homeostasis. ER and other forms of cellular stress also activate anti-oxidative stress genes, which promote cell survival; however, prolonged stress can lead to activation of CCAAT/enhancer-binding protein-homologous protein (CHOP) and caspases to induce cell death (Rasheva and Domingos 2009). Although ER sensing of unfolded proteins is the most common inducer of ER stress, the UPR is activated by multiple stress pathways, including hypoxia and ECM detachment (Avivar-Valderas et al. 2011; Walter and Ron 2011). Stress pathways are major players in multiple pathologies including osteoarthritis (Rasheva and Domingos 2009) and identifying proteins involved in cellular stress pathways may provide new therapeutic targets.

Nuclear factor $\kappa \mathrm{B}(\mathrm{NF} \kappa \mathrm{B} / \mathrm{p} 65 / \mathrm{rel} \mathrm{A})$ is a transcription factor that plays pivotal roles in cell proliferation, differentiation and survival (Marcu et al. 2010). ER stress activates NFKB via tumor necrosis factor- $\alpha$ (TNF- $\alpha)$ receptor associated factor 2 (TRAF2) and inositol requiring enzyme 1 (IRE1) in vitro (Kaneko et al. 2003). The role of NF KB activation in various forms of cellular stress is unclear, but several studies show that NFkB exerts pro-survival functions (Nozaki et al. 2001; Sirabella et al. 2009). In the growth plate, NFKB is essential for chondrocyte viability (Park et al. 2007; Wu et al. 2007). However, the mechanisms that control NFKB activity in the growth plate are unknown.
Autophagy is a lysosomal degradation pathway used to recycle cellular components and activate cell survival under conditions of starvation and stress (Mehrpour et al. 2010). Autophagy is essential for maintaining ER homeostasis, and evidence indicates that during prolonged ER stress, autophagy is activated to assist in the removal of misfolded proteins ( $\mathrm{Li}$ et al. 2008). Crosstalk between cellular stress pathways, autophagy and NFKB-mediated cellular survival has been confirmed previously (Copetti et al. 2009; Sirabella et al. 2009), but whether such crosstalk occurs in chondrocytes is unknown.

The term "matricellular" was coined to encompass a structurally diverse group of proteins that reside in the ECM, but serve no structural functions (Bornstein 2009). Members of the CCN (Cyr61/CTGF/Nov) family of matricellular proteins are critical regulators of cell-ECM interactions. The six members of the $\mathrm{CCN}$ family contain four conserved domains that mediate interactions with integrins, growth factors, and ECM components including heparin sulfate proteoglycans, fibronectin and aggrecan (Jun and Lau 2011). Connective tissue growth factor (CTGF/CCN2) is best known for its role in fibrosis, where its overexpression exacerbates excess collagen deposition in multiple organs (Brigstock 2010). CCN2 overexpression is involved in many other pathological processes, such as atherosclerosis, osteoarthritis and cancer (Chen and Lau 2009).

Although most studies have focused on the mechanisms by which $\mathrm{CCN} 2$ overexpression leads to pathological changes, $\mathrm{CCN} 2$ is indispensible for endochondral bone formation. Global loss of CCN2 in mice results in severe chondrodysplasia and lethality at birth (Ivkovic et al. 2003). This phenotype is accompanied by a significant decrease in chondrocyte proliferation, delayed chondrocyte differentiation, impaired ECM production and insufficient vascular invasion (Hall-Glenn et al. 2012; Ivkovic et al. 2003).

CCN2 induces NFKB activity in ATDC5 chondrocytic cells through integrin $\alpha v \beta 3$-mediated mechanisms to enhance migration (Tan et al. 2009). Reciprocally, the $\mathrm{CCN} 2$ promoter contains an $\mathrm{NF} K \mathrm{~B}$ responsive element that activates $\mathrm{CCN} 2$ expression during the mechanical stretch response in smooth muscle cells (Chaqour et al. 2006). These studies raise the possibility that CCN2 may regulate $\mathrm{NF} K \mathrm{~B}$ in the growth plate. Given the essential role of $\mathrm{NF} K \mathrm{~B}$ in chondrocyte survival, and evidence that $\mathrm{CCN} 2$ induces its expression and activity in multiple cell types, we investigated whether CCN2 plays a role in survival of growth plate chondrocytes. We show that CCN2 is essential for chondrocyte survival, protecting growth plate chondrocytes from proapoptotic pathways activated by cellular stress, and promoting chondrocyte survival. We provide evidence 
consistent with the possibility that $\mathrm{CCN} 2$ enhances chondrocyte survival through activation of $\mathrm{NF} K \mathrm{~B}$ and autophagy. Additionally, we provide evidence that these effects are mediated in part through the ability of CCN2 to engage integrins.

\section{Materials and methods}

Mouse strains and cell lines Ccn2 knockout mice were generated, genotyped and maintained as described (Ivkovic et al. 2003). Ccn2-1- embryos were obtained from timed heterozygote matings, with embryonic day (E) 0.5 representing the detection of a post-copulatory plug.

CCN2 floxed inducible transgenic mice $(C c n 2 f x T g)$ were generated by pronuclear injection as described (Araki et al. 1995; Voncken 2011). These mice were crossed to Col2alCre mice (Ovchinnikov et al. 2000) to induce overexpression of $\mathrm{CCN} 2$ in chondrocytes. Genotyping was performed on DNA isolated from tail biopsies with the following primers: Forward: 5'-TCTTCTGCGATTTCGGCTCC-3'; Reverse: 5'AATGTGTCTTCCAGTCGGTAG-3.' Mouse embryonic fibroblasts (MEFs) from Ccn2fxTg embryos were isolated and cultured as described (Lengner et al. 2004). MEFs were infected for 24 hours with adenoviral Cre-recombinase (AdCRE) and empty vector controls (Ad-CNT) (University of Iowa Gene Transfer Vector Core) at a multiplicity of infection of 300. RNA was isolated using Qiagen RNeasy Kit, and quantitative RT-PCR (qRT-PCR) was used to quantify relative CCN2 expression normalized to glyceraldehyde-3-phosphate dehydrogenase (GAPDH) as described (Kawaki et al. 2008).

Experiments on mice were performed with four sets of WT and Ccn2-/- littermates at E16.5, E18.5 and postnatal day (P) 0 ( $N=8$ per genotype). Animals were treated in accordance with the National Institutes of Health guidelines for care and use of animals, and approved by the UCLA Institutional Animal Care and Use Committee.

Transmission electron microscopy WT and Ccn2-/E18.5 hindlimbs ( $N=3$ per genotype) were prepared for electron microscopy as previously described (Szafranski et al. 2004). The ultrastructural analysis was performed at the Center of Regenerative Medicine for Skeletal Tissues (Department of Clinical Research, University of Bern), in Bern, Switzerland.

Terminal deoxynucleotidyl transferase dUTP nick end labeling (TUNEL) assays TUNEL assays (Roche) were performed on WT and Ccn2-/- growth plates according to the manufacturer's protocol. Cellular counts of chondrocytes were quantitated and normalized to total DAPI counts.
Immunofluorescent staining Embryos were fixed and embedded as described (Ivkovic et al. 2003). $7 \mu \mathrm{m}$ sections were generated using a Lecia GM40 Microtome. For fluorescent staining, Tyramide Signal Amplification was performed according to the manufacturer's protocol (Invitrogen). The following antibodies were used: $\mathrm{CCN} 2$ L-20 (1:1,000; Santa Cruz Biotechnology), BiP (1:500; Cell Signaling), CHOP (1:400; Cell Signaling), Calnexin (1:300; Chemicon), NFkB/RelA/p65 (1:400; Cell Signaling), Apg/Atg12 (1:400; Chemicon) and Apg8b/LC3 (1:200; Abgent). Secondary antibodies were conjugated with AlexaFluor-594 and 488 (Invitrogen). Sections were counterstained with DAPI (Sigma) and mounted with Prolong Gold reagent (Sigma). Immunofluorescence was visualized on an Olympus Bx60 Microscope.

Sternal chondrocyte isolation E16.5 sterna were isolated and pooled by genotype into conical tubes. Garnet bead mix (Invitrogen) was added to $15 \mathrm{ml}$ of HEPES (Sigma) buffered DMEM (HDMEM) (GIBCO). The connective tissue from the sterna was removed by mechanical shaking for $30 \mathrm{~min}$. The media was aspirated, and $0.03 \%$ bacterial collagenase (Chlostridium; Sigma) in HDMEM was added. Additional shaking was performed for $10 \mathrm{~min}$. Cleared sterna were further digested in $0.01 \%$ collagenase overnight. The following day, chondrocytes were filtered $(70 \mu \mathrm{m}$ Fisher) and plated.

Alginate chondron cultures Isolated sternal chondrocytes were suspended in $1.1 \%$ sodium alginate in $1 \mathrm{X}$ Phosphate Buffered Saline (PBS) (Sigma) (Lee et al. 1997). The cell/alginate suspension was extruded drop-wise into $0.1 \mathrm{M}$ Calcium Chloride (Sigma) and beads were polymerized at room temperature for $10-15 \mathrm{~min}$. The beads were then washed with 1X PBS, transferred to T25 flasks and incubated in differentiation media containing $50 \mathrm{ng} / \mathrm{ml}$ ascorbic acid (Sigma). After 7-10 days in culture, the chondrons were released using $10 \mathrm{mM}$ sodium citrate (Sigma). Chondron clusters were plated using Cell-tak adhesive (BD Biosciences) and cultured for 1-3 days for immunofluorescence (Hamamura et al. 2009).

Thapsigargin-induced ER stress in ATDC5 cells ATDC5 chondrosacrcoma cells (RCB0565-Riken Cell Bank) were cultured as described (Altaf et al. 2006). ER stress was induced by a $24 \mathrm{~h}$ treatment with thapsigargin (THG, Sigma) diluted in DMSO at the following concentrations: 0, 40, 80 and 160nM (Hamamura et al. 2009). All experiments were preformed in triplicate and repeated twice.

Integrin blocking and recombinant CCN2 assays Sternal chondrocytes were isolated as described above. Integrin blocking assays were performed as previously described 
(Nishida et al. 2007). Briefly, sternal chondrocytes were plated and serum starved for 6-8 h. Chondrocytes were then treated for $24 \mathrm{~h}$ with an anti-rat integrin $\alpha 5$ blocking antibody (1:100; CD49e BD Pharmingen) or $5 \%$ rat control serum (Sigma). In a separate set of experiments, ATDC5 cells were serum starved followed by a $1 \mathrm{~h}$ treatment with recombinant protein containing only the C terminal (CT) domain of CCN2 $(150 \mathrm{ng} / \mathrm{ml}$; Peprotech) or BSA added to the media, and then by a $1 \mathrm{~h}$ treatment with integrin $\alpha 5$ blocking antibody and/or 80nM THG or DMSO control. Cells were lysed and RNA extracted using a Qiagen RNA purification kit following the manufacturer's instructions. cDNA was generated using Superscript III (Invitrogen). The following primer sequences were used: Integrin alpha 5 Forward: 5'-AGCGCATCTCTCACCATCTT-3' and Reverse: 3'-TCAGGTTCAGTGCGTTCTTGT-5', and normalized to GAPDH as described (Nishida et al. 2007).

CCN2 overexpression in ATDC5 cells ATDC5 cells were infected with a bicistronic adenovirus expressing CCN2 and green florescent protein (AdCCN2-GFP) adenovirus or an adenoviral control (Ad-CNT) vector for $24 \mathrm{~h}$ at a multiplicity of infection of 200 (University of Iowa) (Hall-Glenn et al. 2012). After $24 \mathrm{~h}$, cells were analyzed by immunofluorescence. Experiments were performed in triplicate and repeated twice.

Western blot analysis Proteins were isolated from Ccn2fxTg and WT sterna through lysis in RIPA buffer with $1 \mathrm{X}$ protease (Roche) and $1 \mathrm{X}$ phosphatase inhibitors (Sigma). $30 \mu \mathrm{g}$ of protein lysates were separated by gel electrophoresis and transferred to $0.45 \mu \mathrm{m}$ nitrocellulose membranes (Biorad). Membranes were blocked in milk and incubated at a 1:2,000 dilution of the following primary antibodies overnight at $4{ }^{\circ} \mathrm{C}$ : CCN2 (L-20; Santa Cruz Biotech) and $\beta$-actin (Sigma). The blots were incubated with the following secondary antibodies: Donkey anti-goat horseradish peroxidase (HRP) and Goat anti-rabbit HRP (1:5,000; Biorad). Membranes were developed using Pierce ECL HRP chemoluminescent reagent (ThermoScientific). The blots were repeated twice.

Quantitative reverse transcriptase PCR (qRT-PCR) All qRT-PCR reactions were performed with a SYBR Green Real-time PCR Master Mix (Fermentas) with a Mx3005P QPCR System (Stratagene). Relative expression of Ccn2, Bip and Chop were quantitated and normalized to Gapdh as described and performed in triplicate (Hamamura et al. 2009; Kawaki et al. 2008). Atg12 and Lc3 levels were quantitated and normalized to Gapdh as previously described (Kouroku et al. 2007; Marino et al. 2010).
Statistical analysis

Immunofluorescent quantitation of the levels of $\mathrm{CCN} 2$, BiP and CHOP expression was performed through ImageJ analysis and calculated as a percentage of DAPI positive total cell counts. Three images were taken per independent experiment, followed by quantitation and averaging. At least three independent WT and mutant littermate growth plates were examined with each marker. All in vitro experiments were performed in triplicate and repeated twice. All graphs are represented as fold induction over normalized untreated controls. A normal distribution of the data was assumed, and statistical analysis was performed using Student's $t$-test (95\% confidence interval).

\section{Results}

Loss of CCN2 results in cellular stress in the growth plate

Ccn2 mutant growth plates and cultured chondrocytes exhibit decreased ECM production (Ivkovic et al. 2003; Nishida et al. 2007). However, the consequences on the overall organization of the cartilage ECM were not previously investigated. Therefore, transmission electron microscopy was performed on E18.5 WT and Ccn2 mutant growth plates. Unexpectedly, ultrastructural examination revealed enlarged and distended ERs in proliferating and hypertrophic chondrocytes in Ccn2 mutants (Fig. 1a-d). WT proliferating (Fig. 1a) and hypertrophic (Fig. 1c) chondrocytes contained an organized rough ER (rER) with a limited amount of protein evenly distributed throughout the cisternae. However, in $C c n 2$ mutants, rER cisternae were dilated (Fig. 1b, d). Large vacuoles filled with an electron-lucid granular substance were also observed in mutants, indicative of accumulated intracellular proteins (Fig. 1b, d). Moreover, the nuclear chromatin in mutant chondrocytes was condensed (Fig. 1d), indicating most chondrocytes were undergoing cell death. The mechanism by which chondrocytes undergo physiological cell death is a matter of debate, but condensed chromatin and TUNEL labeling are reliable markers of chondrocyte death (Ahmed et al. 2007). TUNEL staining showed that $\mathrm{Ccn} 2-/-$ growth plates exhibited an increase in the percent of chondrocytes undergoing cell death in both the proliferative and hypertrophic zones compared to WT growth plates (Fig. 1e-g). Ultrastructural analysis also revealed major defects in ECM assembly in mutants. Fewer collagen fibrils were observed throughout the growth plate in Ccn2 mutants compared to WT littermates (Fig. S1).

Because we noted distended ER in proliferative as well as hypertrophic chondrocytes, we examined CCN2 expression and confirmed that $\mathrm{CCN} 2$ protein is expressed both in the 


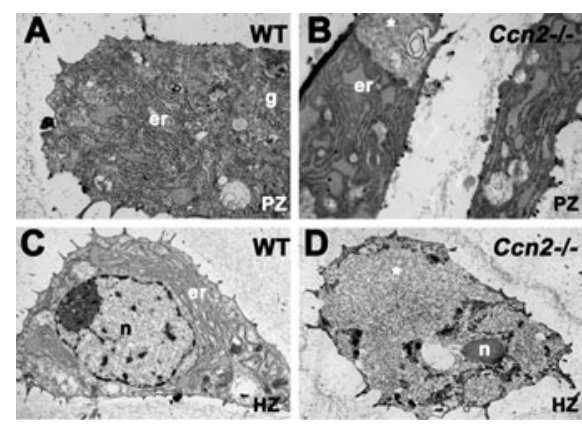

Fig. 1 The Loss of CCN2 results in chondrocyte stress and death. Electron microscopy was performed on WT and Ccn 2 mutant chondrocytes in E18.5 growth plates. a, b WT and Ccn2-/- proliferative chondrocytes, respectively. c, d WT and Ccn2-/- hypertrophic chondrocytes, respectively. The Ccn 2 mutant ER appears devoid of ribosomes and is engorged with an electron lucid substance, which is presumably composed of misfolded protein aggregates. Large vacuoles
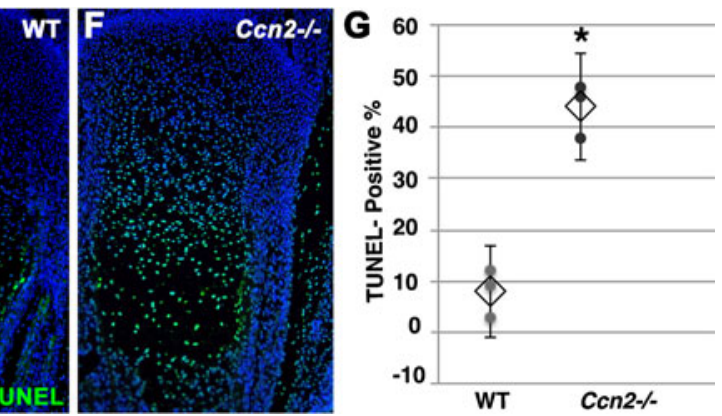

are also present and filled with granulated material (asterisk). e, f WT and $C c n 2$ mutant growth plates analyzed by TUNEL staining showing increased cell death in the proliferative zone in mutants. $g$ Quantitation of TUNEL-positive cells. Open triangles represent the average of the data points. $g$ golgi, $n$ nucleus; $r$ endoplasmic reticulum. Representative images are shown

in the hypertrophic zone (Fig. 2c), and CHOP (Fig. 2e) was undetectable in E16.5 WT growth plates. Increased expression of BiP (Fig. 2d) and CHOP (Fig. 2f) was observed in the hypertrophic zones of $\mathrm{Ccn} 2$ mutant growth plates, but not in the proliferative zone. Expression of calnexin, a calcium dependent ER chaperone, was elevated throughout the proliferative and prehypertrophic zones in $C c n 2$ mutants (Fig. 2g, h). Although calnexin is upregulated by ER stress in chondrocytes (Vranka et al. 2001), the observation that $\mathrm{BiP}$ and $\mathrm{CHOP}$ were not elevated in the proliferative zone in Ccn 2 mutants indicates that these chondrocytes are not undergoing a classical UPR. Nonetheless, these results show that the absence of CCN2 in the growth plate results in defective ECM assembly, increased cellular stress, and chondrocyte cell death in both the proliferative and hypertrophic zones.

\section{$\mathrm{CCN} 2$ Is a stress responsive gene in vitro}

The finding that loss of CCN2 was associated with increased chondrocyte stress raised the possibility that CCN2 may be responsive to cellular stress in chondrocytes. Therefore, we tested whether CCN2 is upregulated in chondrocytes during chemically induced ER stress. Thapsigargin (THG) induces cellular stress by inhibiting sarco/endoplasmic reticulum calcium ATPases, thus blocking calcium release from ER stores (Yang et al. 2005). Prior to cell death, the transcription of ECM components is downregulated and protective ER stress response genes such as BiP are activated by THG in chondrocytes (Yang et al. 2005). ATDC5 chondrocytic cells were treated with increasing concentrations of THG. Bip and Chop levels were examined using qRT-PCR. Consistent with previous studies, THG induced dose-dependent increases in BiP (Fig. 3a) and Chop mRNA (Fig. 3b) (Hamamura et al. 2009). In contrast to the expression of ECM components, which are 


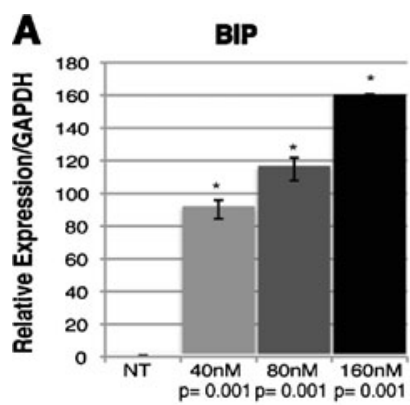

Fig. $3 \mathrm{CCN} 2$ is a stress responsive gene in chemically induced cellular stress. ATDC5 chondrocytes were cultured for $24 \mathrm{~h}$ in the presence of Thapsigargin (THG) at the indicated concentrations. mRNA expression levels of Ccn2 (A), BiP (B) and Chop (c) were assayed by

downregulated by THG (Nugent et al. 2011), Ccn2 mRNA levels were increased by THG with the same kinetics as BiP and Chop (Fig. 3c), suggesting that CCN2 is a stressresponsive gene in chondrocytes in vitro.

CCN2 depletion induces cellular stress in chondron cultures

The above experiments demonstrate that $\mathrm{CCN} 2$ expression is induced by stress in vitro. However, they do not demonstrate that the cellular stress observed in Ccn2-/- growth plates is due to a direct role for CCN2 in chondrocytes. Recent studies have shown that the presence of ECM protects chondrocytes from ER stress (Nugent et al. 2011). Therefore, a 3-dimensional culture system was developed in which chondrocytes were maintained in alginate, yielding chondroctye clusters (chondrons) (Fig. S2) (Lee et al. 1997). The accumulation of ECM components can be observed (Fig. S2A, B). THG treatment induced cellular stress in chondrons, with BiP localization transitioning from the ER lumen in control cells (Fig. S2C), to the nucleus/perinuclear region in THG treated chondrons (Fig. S2D). The relocalization of $\mathrm{BiP}$ to the nucleus has not been reported previously in chondrocytes, but is consistent with previous reports in other cell types (Ni et al. 2011). CHOP levels were also increased in THG treated chondrons (Fig. S2F), compared to untreated controls (Fig. S2E). Therefore, chondrons in alginate are sensitive to THGinduced stress. Next, the impact of loss of Ccn2 on THG-induced stress was tested. First, we confirmed that CCN2 is expressed in WT chondrons (Fig. 4a) and is absent in Ccn $2-1-$ chondrons (Fig. 4b, c). WT chondrons expressed low levels of BiP (Fig. 4d). Ccn2 -/- chondrons displayed upregulated BiP expression and a higher proportion of nuclear BiP-positive cells (Fig. 4e, f). CCN2 mutant chondrons also exhibit increased expression of CHOP (Fig. 4g-i). These results suggest that $\mathrm{CCN} 2$ plays a direct role in the protection of chondrocytes against THG-induced stress in vitro.

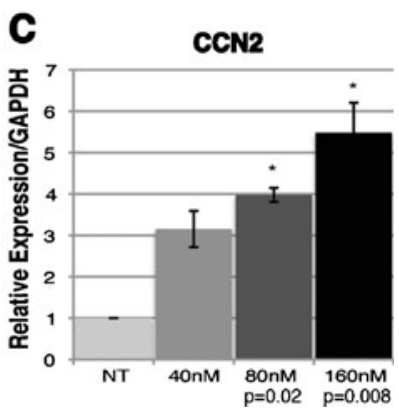

Quantitative Reverse Transcriptase PCR (qRT-PCR). Assays were performed in triplicate and a $p$ value of $>0.05$ was considered significant compared to no THG (*). $p$ values shown are for $160 \mathrm{nM}$ THG

The protective effect of $\mathrm{CCN} 2$ against chondrocyte stress may be mediated through integrin $\alpha 5$

$\mathrm{CCN} 2$ is thought to mediate its functions by acting as a ligand for integrins (Jun and Lau 2011). CCN2 induces expression of integrin $\alpha 5$, and binds to integrin $\alpha 5 \beta 1$ to promote chondrocyte proliferation and ECM secretion (Nishida et al. 2007). Integrin $\alpha 5 \beta 1$ is also required for chondrocyte survival (Pulai et al. 2002). Recent data demonstrate that multiple stress pathways are activated as a survival mechanism during cell detachment from the ECM (Avivar-Valderas et al. 2011). Taken together, these findings suggest that one of the prosurvival functions of integrin $\alpha 5 \beta 1$ in chondrocytes may be the suppression of cellular stress. To test this possibility, sternal chondrocytes were treated with or without an integrin $\alpha 5$-blocking antibody for $24 \mathrm{~h}$ (Fig. 5a). BiP and Chop mRNA expression levels were upregulated in response to treatment with the integrin $\alpha 5$-blocking antibody (Fig. 5a). Consistent with our findings that loss of $\mathrm{Ccn} 2$ leads to increased cellular stress in chondron cultures (Fig. 4), recombinant C-terminal CCN2 (rCCN2)-treated ATDC5 cells exhibited a decrease in Chop mRNA levels compared to BSA treated controls in the presence of THG (Fig. 5b). The C-terminal domain of $\mathrm{rCCN} 2$ contains the integrin $\alpha 5 \beta 1$ binding domain (Gao and Brigstock 2006). Next, ATDC5 cells were pre-treated for $1 \mathrm{~h}$ with recombinant $\mathrm{C}$-terminal rCCN2 added to the medium prior to stress induction for $1 \mathrm{~h}$ in the presence of the $\alpha 5$-blocking antibody. As in Fig. 5a, treatment with integrin $\alpha 5$-blocking antibody led to increased Chop mRNA expression (Fig. 5c, columns 1 and 2), whereas treatment of ATDC5 cells with THG leads to an approximately 2-fold induction in Chop mRNA (Fig. 5b, columns 1 and 2). Also, treatment with THG in the presence of the integrin $\alpha 5$-blocking antibody leads to an approximately 10 -fold induction of Chop (Fig. 5c, columns 1 and 3). Moreover, while rCCN2 prevented THG-induced Chop expression (Fig. 5b, columns 2 and 4), treatment with integrin $\alpha 5$-blocking antibody prevented the protective effect of rCCN2 (Fig. 5c, 

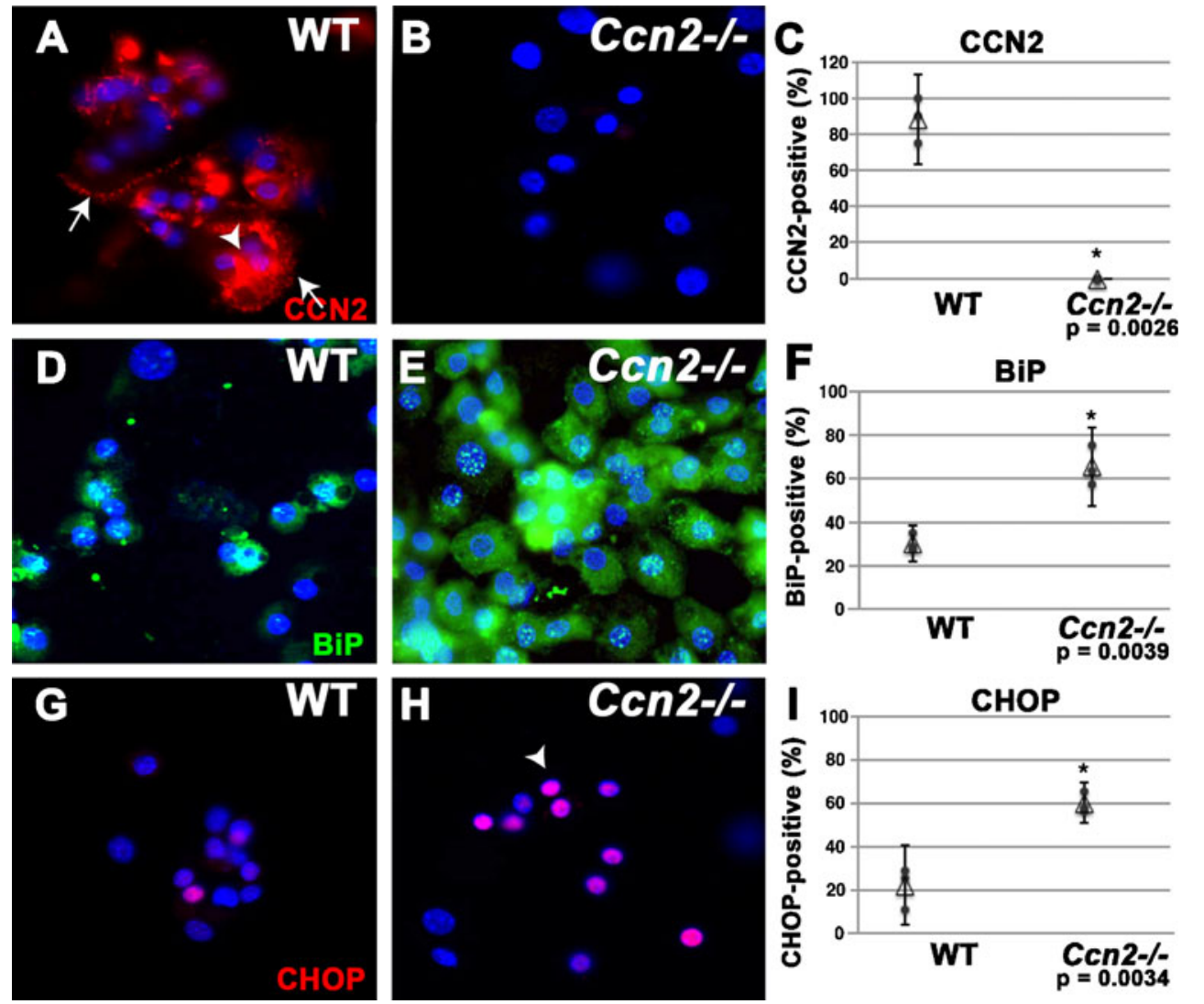

Fig. 4 CCN2 depleted chondrons exhibit elevated cellular stress. Primary sternal chondrocytes were isolated from E16.5 WT and Ccn2 -/ - sterna, and cultured in alginate for 10 days to yield chondrons. $\mathrm{CCN} 2, \mathrm{BiP}$ and $\mathrm{CHOP}$ protein expression were analyzed through immunofluorescence. a-c CCN2 expression. $\mathrm{CCN} 2$ protein expression was abundant in WT chondrons (a), where punctate CCN2 protein expression was observed intracellularly (arrowhead) and pericellularly (arrows). CCN2 protein was not detected in Ccn2 mutant chondrons. b, c Quantitation of number of CCN2-expressing cells. d-f BiP expression in WT and Ccn $2-/-$ chondrons. $\mathbf{d ~ B i P}$ is detected in the ER in some cells in WT chondron clusters. e Nearly all Ccn2-/- chondrocytes express $\mathrm{BiP}$, and many nuclei are positive. f Quantitation of the percentage of nuclear BiP-expressing cells. g-i CHOP expression. g Few WT chondrocytes express CHOP. In contrast, most Ccn2-/ - chondrocytes express CHOP $(\mathrm{H})$. i Quantitation of the percentage of CHOP-expressing chondrocytes. c, f, i CCN2, BiP, and CHOP expression was analyzed in triplicate using ImageJ analysis and calculated as a percentage of DAPI positive total cell counts. Asterisks indicate statistical significance with a $p$-value of 0.003 . Open triangles in (c), (f), and (i) represent the averages of the data points. Representative images are shown
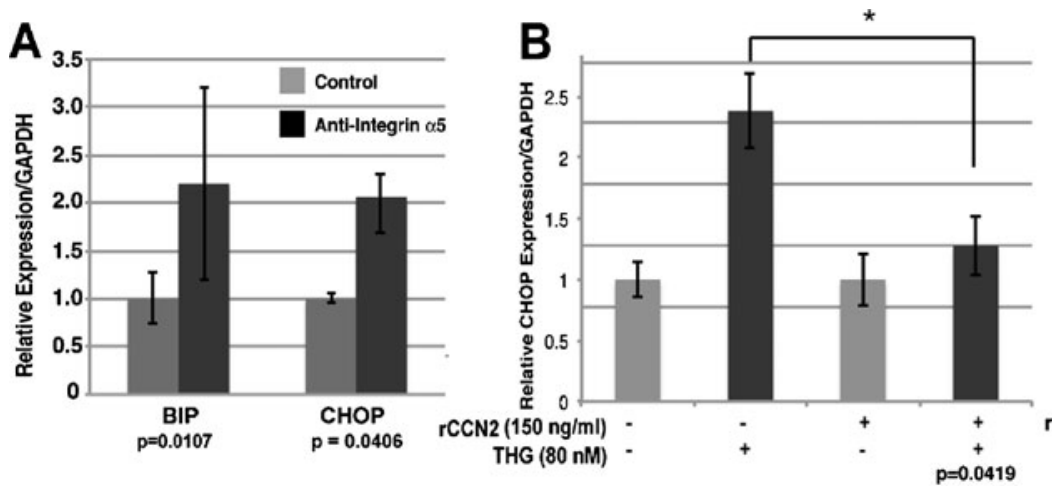

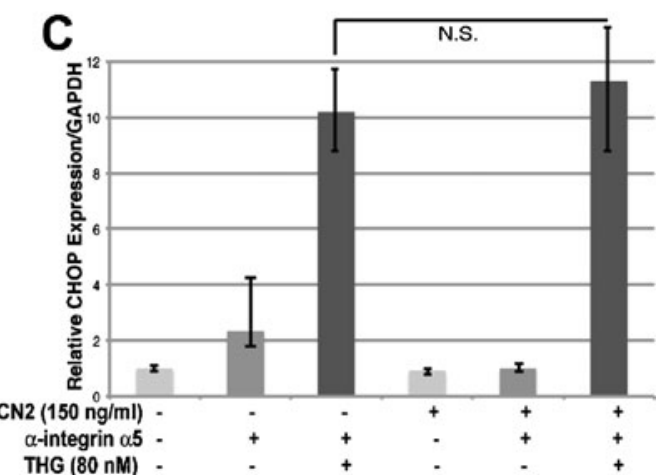

Fig. 5 Blocking Integrin $\alpha 5$ results in chondrocyte stress. Primary sternal chondrocytes were serum starved and treated with anti-integrin $\alpha 5$ blocking antibody or goat serum (control) for $24 \mathrm{~h}$, after which BiP and CHOP mRNA levels were quantitated by qRT-PCR and normalized to GAPDH. BiP and CHOP expression were increased by treatment with the blocking antibody. b $\mathrm{rCCN} 2$ protects against THG-induced stress, resulting in Chop mRNA induction. c This affect is attenuated upon treatment with $\alpha 5$ blocking antibody and THG compared to DMSO and rat serum controls 
columns 3 and 6). These results, although preliminary, suggest that integrin $\alpha 5$ may be required for the ability of CCN2 to protect against cellular stress in chondrocytes in vitro.

Overexpression of CCN2 attenuates chondrocyte stress

Since loss of CCN2 induces cellular stress in cartilage, we generated transgenic mice that overexpress $\mathrm{CCN} 2$ in chondrocytes to determine whether increased $\mathrm{CCN} 2$ could lead to reduced stress. An inducible transgenic construct was generated, consisting of a floxed transcriptional STOP signal placed upstream of the $C c n 2$ coding sequence (Fig 6a). Transgenic lines were established and mated to Col2Cre mice (Ovchinnikov et al. 2000) to induce chondrocyte-specific overexpression of CCN2 (Fig 6a). A 3-5 fold induction of CCN2 protein was observed in Ccnfx2 Tg;Col2Cre mice (Fig. 6b). The specificity of induction was confirmed in isolated mouse embryonic fibroblasts (MEFs) from Ccn2fxTg embryos infected with adenoviral Cre (Ad-CRE) (Fig. 6c). Ccn2fxTg; Col2Cre mice were viable with no obvious morphological differences at birth
(Fig. 6d), but exhibited progressive overgrowth of cartilage elements (data not shown). A complete characterization of these transgenics will be presented elsewhere. The growth plates of P0 Ccn $2 f x T g$ tibiae had normal chondrocyte organization, although both the hypertrophic zone and chondrocytes within it were slightly smaller than in WT littermates (Fig. 6e, f). Consistent with a protective function for $\mathrm{CCN} 2$, levels of $\mathrm{BiP}$ were reduced in $\mathrm{Ccn} 2 \mathrm{fx} \mathrm{Tg}$; Col2Cre mice compared to WT controls (Fig. 6g, h).

\section{CCN2 regulates NF $\kappa B$ expression and autophagy}

NFKB is required for chondrocyte survival (Park et al. 2007; $\mathrm{Wu}$ et al. 2007). CCN2 induces nuclear localization of NFKB and activates pro-survival mechanisms, while inhibiting stress-mediated apoptosis in a number of cell types (Gao and Brigstock 2005). NFkB is activated by integrin $\alpha 5 \beta 1$ in breast cancer epithelial cells (Sen et al. 2010) and $\alpha 5 \beta 1$ is a major receptor for CCN2 in chondrocytes (Sen et al. 2010). Our earlier results suggested that $\mathrm{CCN} 2$ protects against chondrocyte stress through

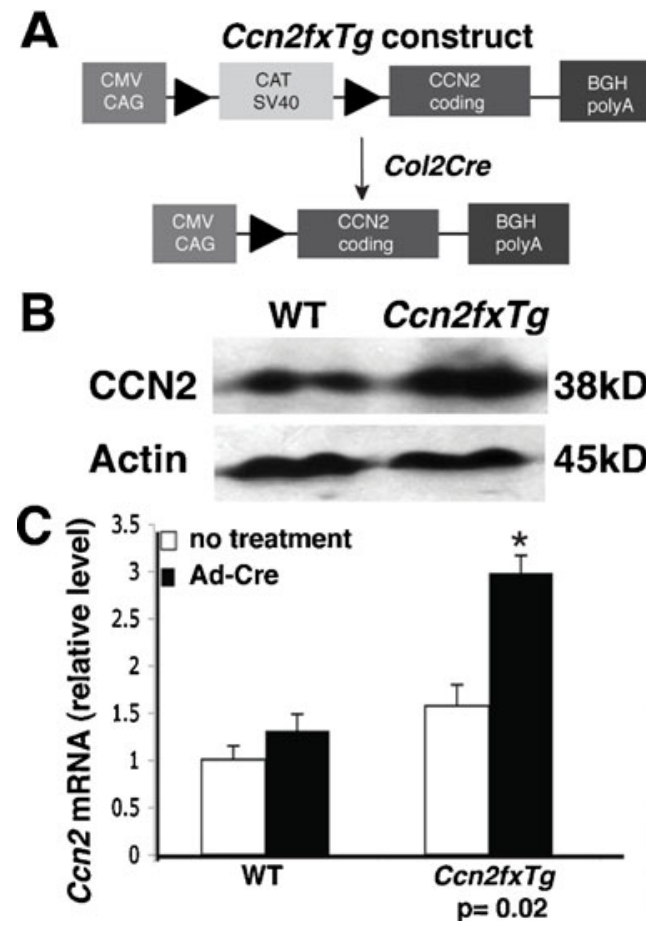

Fig. 6 CCN2 overexpression attenuates ER stress in vivo. a Schematic of the CCN2 floxed transgenic construct. A floxed cassette consisting of a STOP cassette and a chloroacetyltransferase (CAT) gene is flanked by loxP sites and inserted between, the $\mathrm{CMV} /$ chicken $\beta$ actin (CAG) promoter and the murine $\mathrm{CCN} 2$ coding sequence, followed by a bovine growth hormone polyA signal sequence. Upon crossing to a Col2Cre mouse, the silencer is excised, leading to transcription of CCN2 from the transgene in cartilage. $\mathbf{b}$ Western blot of protein isolated from sterna of WT and Ccn2fxTg;Col2Cre P0 pups with actin controls. c Ccn2 mRNA levels in mouse embryonic fibroblasts (MEFs) from E11.5
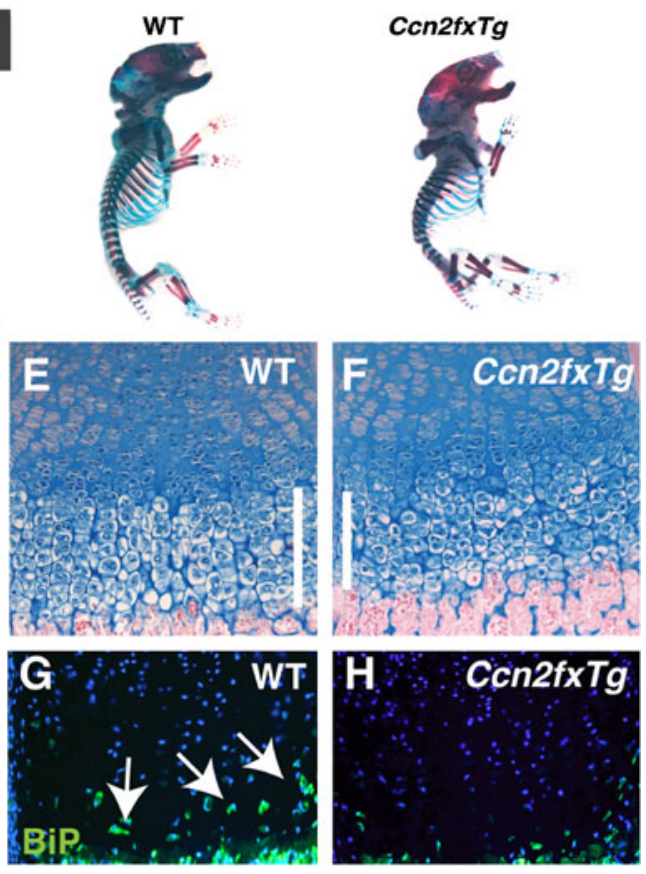

CCN2fxtg E11.5 embryos infected with AdCre or Adcontrol. Ccn2 mRNA levels are not significantly different in WT vs. Ccn2fxTg MEFs in the absence of Cre, but are increased approximately 2.5 -fold in Ccn2fxTg mice in the presence of Cre. (D) Skeletal preparations of postnatal day 0 (P0) Ccn2fxTg;Col2Cre and WT littermates, showing no apparent morphological differences. e, f Growth plate histology of P0 WT (e) and Ccn2fxTg;Col2Cre (f) littermate. The hypertrophic zone is slightly shorter and hypertrophic chondrocytes are smaller in transgenics. $\mathbf{g}$, h Overexpression of Ccn2 results in decreased BiP expression (h) compared to WT littermate (g) in P0 growth plates 
integrin $\alpha 5$ in vitro (Fig. 5). We therefore investigated whether CCN2 might exert pro-survival functions in chondrocytes at least in part through regulation of NFKB. NF $\kappa B$ localization was examined in E16.5 WT and mutant growth plates. In WT proliferative zones, NFKB was predominantly localized to the nucleus (Fig. 7a), similar to previous reports (Park et al. 2007; Wu et al. 2007). In Ccn2-/- growth plates, NFKB was present in the cytoplasm, but the number of cells exhibiting nuclear localization was decreased (Fig. 7b,c). These data are thus consistent with previous in vitro studies showing that $\mathrm{CCN} 2$ induces $\mathrm{NFKB}$ activation (Gao and Brigstock 2005), and raise the possibility that $\mathrm{CCN} 2$ exerts pro-survival effects in chondrocytes at least in part through NFKB.

Autophagy is an important survival mechanism in normal chondrocytes and in response to stress (Lotz and Carames 2011). We examined the expression of autophagosome markers Atg12 and LC3 (Atg8), which conjugate with one another to stabilize autophagosome formation (Mehrpour et al. 2010). In E16.5 WT growth plates, Atg12 and LC3 were expressed throughout the growth plate, with the highest levels in the hypertrophic zone (Fig. 7d, f), consistent with previous literature showing that autophagy is required for terminal chondrocyte differentiation (Srinivas et al. 2009). However, in Ccn2-/- growth plates Atg12 and LC3 protein (Fig. 7e,g) and mRNA (Fig. 7h, i) levels were decreased.

Because NFKB and autophagy have pro-survival functions in chondrocytes, we investigated whether CCN2 can induce NFKB and the expression of autophagy genes. ATDC5 cells infected with a CCN2 overexpressing adenovirus (Ad-CCN2-GFP) exhibited increased $\mathrm{CCN} 2$ expression within $24 \mathrm{~h}$ after infection (Fig. 8a, b). Consistent with in vivo studies (Fig. 7), $\mathrm{CCN} 2$ expression induced nuclear $\mathrm{NFKB}$ expression (Fig. 8c, d). Furthermore, CCN2 stimulated the expression of LC3 (Fig. 8e, f). These data suggest that CCN2 may protect against chondrocyte stress by upregulating pro-survival NFKB and autophagy pathways.
Fig. 7 Decreased NFKB and autophagy-mediated cell survival in $C c n 2$ mutants. $\mathrm{NF} \kappa \mathrm{B}$ expression was visualized by immunofluorescence in E16.5 WT and $C c n 2$ mutant growth plates. a In WT growth plates, $\mathrm{NF} \kappa \mathrm{B}$ was primarily localized to the nucleus throughout the proliferative zone. b In Ccn2-/ - growth plates, NFKB expression in the proliferative zone was decreased and localized mainly to the cytoplasm. c NFKB nuclear expression throughout the growth plate was quantitated using Image $J$ analysis as the percentage of DAPI positive nuclei. Growth plate immunostaining was performed in triplicate; asterisk denotes a $p$-value of 0.003 , indicating statistical significance. Open triangle indicates the average of the data points. d, e E16.5 WT (D) and Ccn2-/- (e) growth plates immunostained for Atg12. f, g E16.5 WT (F) and Ccn2-/- (g) growth plates immunostained for Atg8 (LC3). All sections were counterstained with DAPI. Stains were performed at least twice and representative images are shown. $\mathbf{h}, \mathbf{i} \operatorname{Atg} 12$ and $L C 3$ mRNA levels were quantitated by qRT-PCR in WT and Ccn2-/ - sternal chondrocytes
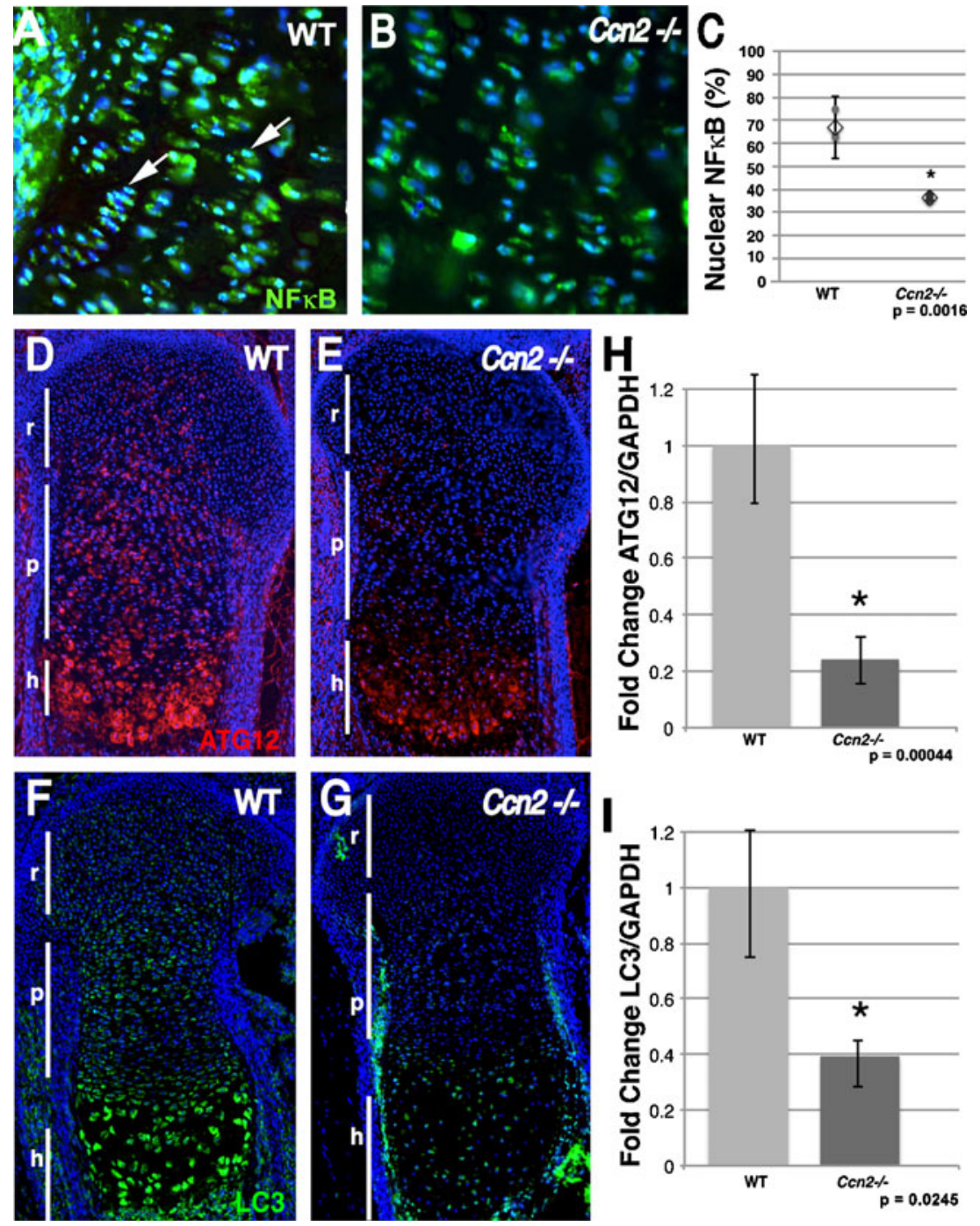

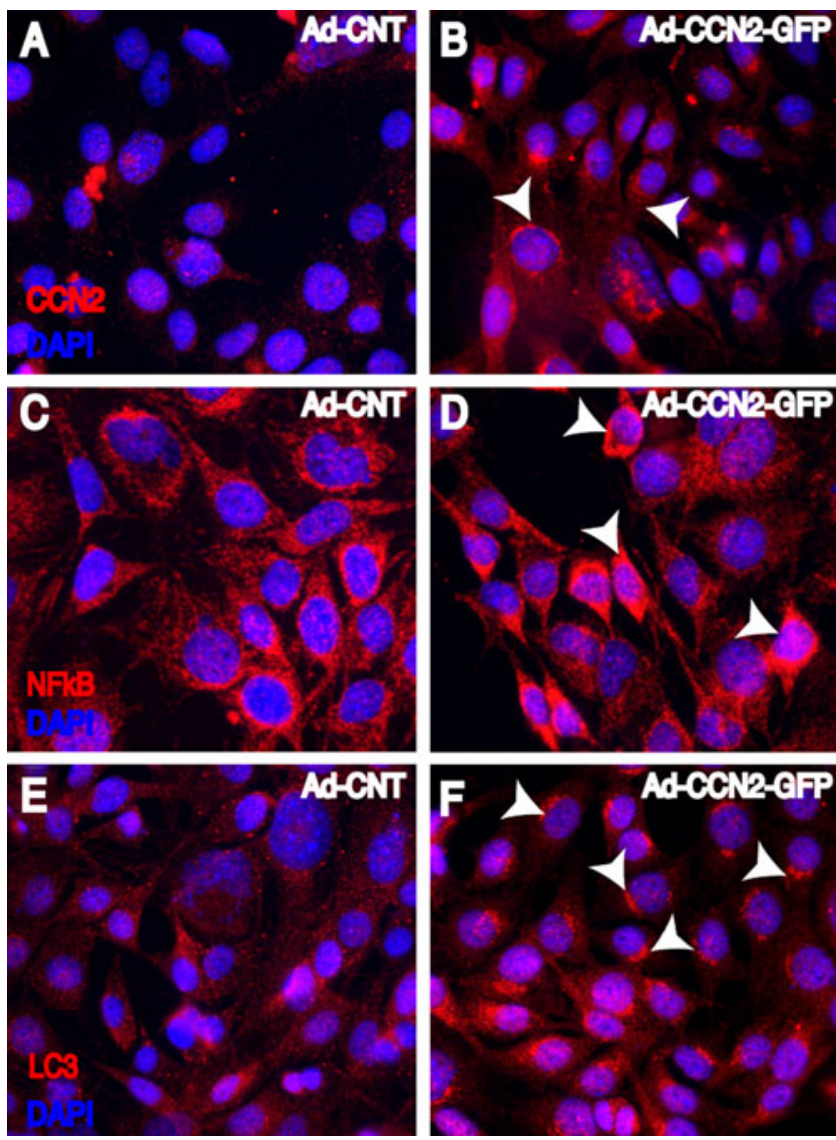

Fig. 8 Ectopic CCN2 expression results in increased NFKB and expression of autophagy genes in ATDC5 cells. NFKB and autophagy marker LC3 were examined in ATDC5 cells infected with Ad-CCN2green florescent protein (Ad-CCN2-GFP) and Ad-control (Ad-CNT) constructs. a, b CCN2 expression is upregulated in Ad-CCN2-GFP ATDC5 cells (b) compared to Ad-CNT treated cells (a). c, d Nuclear NFKB is increased in Ad-CCN2-GFP cells (d) compared to control cells (c). e, $\mathbf{f}$ Expression of LC3/Atg8 is increased in Ad-CCN2-GFP cells (f) compared to Ad-CNT treated cells (e). (Red-CCN2, NFkB and LC3); (Blue = DAPI counterstain). Representative images are shown

\section{Discussion}

The data reported here provide evidence that $\mathrm{CCN} 2$ is important for ECM assembly and chondrocyte survival in the growth plate. Ultrastructural analysis revealed that loss of CCN2 leads to defective ECM organization and chondrocyte death, accompanied by increased cellular stress. The activation of stress pathways in vivo was confirmed by elevated levels of $\mathrm{BiP}$ and $\mathrm{CHOP}$ in $C \mathrm{cn} 2-1-$ growth plates. These results were recapitulated in vitro, where loss of $\mathrm{Ccn} 2$ in chondron cultures also resulted in cellular stress. This finding suggests that the role of $\mathrm{CCN} 2$ in protecting chondrocytes from stress is direct, rather than an indirect consequence of defective growth plate angiogenesis. The finding that $C c n 2$ expression is induced by stress, unlike structural ECM proteins, whose expression is downregulated, suggests that an important function of $\mathrm{CCN} 2$ may be to mediate chondrocyte survival.

The precise mechanisms by which $\mathrm{CCN} 2$ protects against cellular stress remain unclear. Integrin $\alpha 5 \beta 1$ is essential for chondrocyte survival in vitro (Pulai et al. 2002). Integrin $\alpha 5$ expression was decreased in Ccn2-/chondrocytes, leading to defective chondrocyte adhesion and downstream integrin signaling (Nishida et al. 2007). Evidence that CCN2 may exert a pro-survival effect through modulation of cell-ECM attachment comes from the finding that treatment of chondrocytes with an integrin $\alpha 5$-blocking antibody leads to increased cellular stress, while treatment of ATDC5 cells with C-terminal rCCN2 (which contains the $\alpha 5 \beta 1$ binding site) (Gao and Brigstock 2006) mediates a modest protective effect during chemically induced stress, which is attenuated in the presence of an integrin $\alpha 5$-blocking antibody. Currently, no in vivo studies have been conducted to assess the role of $\alpha 5$ containing integrins in cartilage. However, the loss of $\beta 1$ integrin leads to reduced ECM production, growth plate disorganization and impaired survival (Aszodi et al. 2003), consistent with a potential role for $\alpha 5 \beta 1$ in ECM organization and chondrocyte survival.

In addition to induction of pro-apoptotic stress pathways, the loss of $C c n 2$ also results in decreased expression of nuclear NFKB and autophagy genes in vivo. In accordance, overexpression of $\mathrm{Ccn} 2$ in chondrocytes resulted in induction of nuclear NFKB and autophagy gene expression. A limitation of these experiments is that the ability of overexpression of $\mathrm{CCN} 2$ to rescue NFKB and autophagy gene expression in Ccn2-/chondrocytes was not tested in vitro. Thus, we cannot determine whether the effect of CCN2 is direct or indirect. However, both $\mathrm{NFKB}$ and autophagy exert pro-survival functions in growth plate chondrocytes (Srinivas et al. 2009; Wu et al. 2007). The mechanism by which $\mathrm{CCN} 2$ induces the expression of $\mathrm{NFKB}$ in chondrocytes is unknown but probably occurs through integrin signaling, as previously observed in other cell types (Gao and Brigstock 2005; Zahir et al. 2003).

$\mathrm{CCN} 2$ expression is correlated with the onset of osteoarthritis (OA) and synovial damage (Blaney Davidson et al. 2006; Omoto et al. 2004). Paradoxically, recombinant CCN2 expedites repair of articular cartilage during chemically induced knee OA (Nishida et al. 2004). These opposing effects of CCN2 may be due to its ability to modulate NFkB. While NFkB has pro-survival functions in chondrocytes (Park et al. 2007; Wu et al. 2007), it also promotes the expression of pro-inflammatory genes in OA chondrocytes (Marcu et al. 2010). Whether NFKB has a prosurvival function in OA chondrocytes is unknown. Further investigations of the mechanism by which $\mathrm{CCN} 2$ promotes 
survival in growth plate chondrocytes, and its role in the maintenance of articular cartilage may provide new avenues to treat and prevent $\mathrm{OA}$.

Acknowledgments We thank Jeannine Wagner for expert technical assistance. This work was supported by grants: F31 AR060687-01 (FHG), R01 AR052686 (KML), Beckman Foundation (JRO), and the Swiss National Science Foundation (EBH).

Author contributions KML, PB, EBH and FHG were involved in the conception and design of the experiments. FHG, AD, AA, LA, RRB, JRO and EBH performed the experiments and analyzed the data. FFS, FvN and RG generated crucial reagents. FHG and KML wrote the article.

Open Access This article is distributed under the terms of the Creative Commons Attribution License which permits any use, distribution, and reproduction in any medium, provided the original author(s) and the source are credited.

\section{References}

Ahmed YA, Tatarczuch L, Pagel CN, Davies HM, Mirams M, Mackie EJ (2007) Physiological death of hypertrophic chondrocytes. Osteoarthr Cartil OARS Osteoarthr Res Soc 15(5):575-586

Altaf FM, Hering TM, Kazmi NH, Yoo JU, Johnstone B (2006) Ascorbate-enhanced chondrogenesis of ATDC5 cells. Eur Cell Mater 12:64-69, discussion 69-70

Araki K, Araki M, Miyazaki J, Vassalli P (1995) Site-specific recombination of a transgene in fertilized eggs by transient expression of Cre recombinase. Proc Natl Acad Sci U S A 92(1):160-164

Aszodi A, Hunziker EB, Brakebusch C, Fassler R (2003) Beta1 integrins regulate chondrocyte rotation, G1 progression, and cytokinesis. Genes Dev 17(19):2465-2479

Avivar-Valderas A, Salas E, Bobrovnikova-Marjon E, Diehl JA, Nagi C, Debnath J, Aguirre-Ghiso JA (2011) PERK integrates autophagy and oxidative stress responses to promote survival during extracellular matrix detachment. Mol Cell Biol 31(17):3616-3629

Barbieri O, Astigiano S, Morini M, Tavella S, Schito A, Corsi A, Di Martino D, Bianco P, Cancedda R, Garofalo S (2003) Depletion of cartilage collagen fibrils in mice carrying a dominant negative Col2a1 transgene affects chondrocyte differentiation. Am J Physiol Cell Physiol 285(6):C1504-C1512

Bateman JF, Boot-Handford RP, Lamande SR (2009) Genetic diseases of connective tissues: cellular and extracellular effects of ECM mutations. Nature reviews. Genetics 10(3):173-183

Bertolotti A, Zhang Y, Hendershot LM, Harding HP, Ron D (2000) Dynamic interaction of BiP and ER stress transducers in the unfolded-protein response. Nat Cell Biol 2(6):326-332

Blaney Davidson EN, Vitters EL, Mooren FM, Oliver N, Berg WB, van der Kraan PM (2006) Connective tissue growth factor/CCN2 overexpression in mouse synovial lining results in transient fibrosis and cartilage damage. Arthritis Rheum 54(5):1653-1661

Bornstein P (2009) Matricellular proteins: an overview. J Cell Commun Signal 3(3-4):163-165

Brigstock DR (2010) Connective tissue growth factor (CCN2, CTGF) and organ fibrosis: lessons from transgenic animals. J Cell Commun Signal 4(1):1-4

Chaqour B, Yang R, Sha Q (2006) Mechanical stretch modulates the promoter activity of the profibrotic factor $\mathrm{CCN} 2$ through increased actin polymerization and NF-kappaB activation. J Biol Chem 281(29):20608-20622
Chen CC, Lau LF (2009) Functions and mechanisms of action of CCN matricellular proteins. Int J Biochem Cell Biol 41(4):771-783

Copetti T, Bertoli C, Dalla E, Demarchi F, Schneider C (2009) p65/ RelA modulates BECN1 transcription and autophagy. Mol Cell Biol 29(10):2594-2608

Gao R, Brigstock DR (2005) Activation of nuclear factor kappa B (NFkappaB) by connective tissue growth factor (CCN2) is involved in sustaining the survival of primary rat hepatic stellate cells. Cell Commun Signal 3:14

Gao R, Brigstock DR (2006) A novel integrin alpha5beta1 binding domain in module 4 of connective tissue growth factor (CCN2) CTGF) promotes adhesion and migration of activated pancreatic stellate cells. Gut 55(6):856-862

Hall-Glenn F, De Young RA, Huang BL, van Handel B, Hofmann JJ, Chen TT, Choi A, Ong JR, Benya PD, Mikkola H, Iruela-Arispe ML, Lyons KM (2012) CCN2/connective tissue growth factor is essential for pericyte adhesion and endothelial basement membrane formation during angiogenesis. PLoS One 7(2):e30562

Hamamura K, Goldring MB, Yokota H (2009) Involvement of p38 MAPK in regulation of MMP13 mRNA in chondrocytes in response to surviving stress to endoplasmic reticulum. Arch Oral Biol 54(3):279-286

Ivkovic S, Yoon BS, Popoff SN, Safadi FF, Libuda DE, Stephenson RC, Daluiski A, Lyons KM (2003) Connective tissue growth factor coordinates chondrogenesis and angiogenesis during skeletal development. Development 130(12):2779-2791

Jun JI, Lau LF (2011) Taking aim at the extracellular matrix: CCN proteins as emerging therapeutic targets. Nat Rev Drug Discov 10(12):945-963

Kaneko M, Niinuma Y, Nomura Y (2003) Activation signal of nuclear factor-kappa B in response to endoplasmic reticulum stress is transduced via IRE1 and tumor necrosis factor receptorassociated factor 2. Biol Pharm Bull 26(7):931-935

Kawaki H, Kubota S, Suzuki A, Lazar N, Yamada T, Matsumura T, Ohgawara T, Maeda T, Perbal B, Lyons KM, Takigawa M (2008) Cooperative regulation of chondrocyte differentiation by $\mathrm{CCN} 2$ and $\mathrm{CCN} 3$ shown by a comprehensive analysis of the CCN family proteins in cartilage. J Bone Miner Res 23(11):1751-1764

Kouroku Y, Fujita E, Tanida I, Ueno T, Isoai A, Kumagai H, Ogawa S, Kaufman RJ, Kominami E, Momoi T (2007) ER stress (PERK/ eIF2alpha phosphorylation) mediates the polyglutamine-induced LC3 conversion, an essential step for autophagy formation. Cell Death Differ 14(2):230-239

Lee GM, Poole CA, Kelley SS, Chang J, Caterson B (1997) Isolated chondrons: a viable alternative for studies of chondrocyte metabolism in vitro. Osteoarthr Cartil 5(4):261-274

Lengner CJ, Lepper C, van Wijnen AJ, Stein JL, Stein GS, Lian JB (2004) Primary mouse embryonic fibroblasts: a model of mesenchymal cartilage formation. J Cell Physiol 200(3):327-333

Li J, Ni M, Lee B, Barron E, Hinton DR, Lee AS (2008) The unfolded protein response regulator GRP78/BiP is required for endoplasmic reticulum integrity and stress-induced autophagy in mammalian cells. Cell Death Differ 15(9):1460-1471

Lotz MK, Carames B (2011) Autophagy and cartilage homeostasis mechanisms in joint health, aging and OA. Nat Rev Rheumatol 7(10):579-587

Marcu KB, Otero M, Olivotto E, Borzi RM, Goldring MB (2010) NFkappaB signaling: multiple angles to target OA. Current Drug Targets 11(5):599-613

Marino G, Fernandez AF, Cabrera S, Lundberg YW, Cabanillas R, Rodriguez F, Salvador-Montoliu N, Vega JA, Germana A, Fueyo A, Freije JM, Lopez-Otin C (2010) Autophagy is essential for mouse sense of balance. J Clin Investig 120(7):2331-2344

Mehrpour M, Esclatine A, Beau I, Codogno P (2010) Overview of macroautophagy regulation in mammalian cells. Cell Res 20(7):748-762 
Ni M, Zhang Y, Lee AS (2011) Beyond the endoplasmic reticulum: atypical GRP78 in cell viability, signalling and therapeutic targeting. Biochem J 434(2):181-188

Nishida T, Kubota S, Kojima S, Kuboki T, Nakao K, Kushibiki T, Tabata Y, Takigawa M (2004) Regeneration of defects in articular cartilage in rat knee joints by CCN2 (connective tissue growth factor). J Bone Miner Res 19(8):1308-1319

Nishida T, Kawaki H, Baxter RM, Deyoung RA, Takigawa M, Lyons KM (2007) CCN2 (Connective Tissue Growth Factor) is essential for extracellular matrix production and integrin signaling in chondrocytes. J Cell Commun Signal 1(1):45-58

Nozaki S, Sledge GW Jr, Nakshatri H (2001) Repression of GADD153/CHOP by NF-kappaB: a possible cellular defense against endoplasmic reticulum stress-induced cell death. Oncogene 20(17):2178-2185

Nugent AE, McBurney DL, Horton WE Jr (2011) The presence of extracellular matrix alters the chondrocyte response to endoplasmic reticulum stress. J Cell Biochem 112(4):1118-1129

Omoto S, Nishida K, Yamaai Y, Shibahara M, Nishida T, Doi T, Asahara $\mathrm{H}$, Nakanishi T, Inoue H, Takigawa M (2004) Expression and localization of connective tissue growth factor (CTGF/Hcs24/ CCN2) in osteoarthritic cartilage. Osteoarthr Cartil 12(10):771-778

Ovchinnikov DA, Deng JM, Ogunrinu G, Behringer RR (2000) Col2a1-directed expression of Cre recombinase in differentiating chondrocytes in transgenic mice. Genesis 26(2):145-146

Park M, Yong Y, Choi SW, Kim JH, Lee JE, Kim DW (2007) Constitutive RelA activation mediated by Nkx3.2 controls chondrocyte viability. Nat Cell Biol 9(3):287-298

Pulai JI, Del Carlo M Jr, Loeser RF (2002) The alpha5beta1 integrin provides matrix survival signals for normal and osteoarthritic human articular chondrocytes in vitro. Arthritis Rheum 46(6):1528-1535

Rasheva VI, Domingos PM (2009) Cellular responses to endoplasmic reticulum stress and apoptosis. Apoptosis 14(8):996-1007

Sen T, Dutta A, Chatterjee A (2010) Epigallocatechin-3-gallate (EGCG) regulates gelatinase-B (MMP-9) by involvement of FAK/ERK/NFkappaB and AP-1 in the human breast cancer cell line MDA-MB-231. Anti-cancer drugs 21(6):632-644

Sirabella R, Secondo A, Pannaccione A, Scorziello A, Valsecchi V, Adornetto A, Bilo L, Di Renzo G, Annunziato L (2009) Anoxiainduced NF-kappaB-dependent upregulation of NCX1 contributes to $\mathrm{Ca} 2+$ refilling into endoplasmic reticulum in cortical neurons. Stroke J Cereb Circ 40(3):922-929
Srinivas V, Bohensky J, Shapiro IM (2009) Autophagy: a new phase in the maturation of growth plate chondrocytes is regulated by HIF, mTOR and AMP kinase. Cells Tissues Organs 189(1-4):88-92

Szafranski JD, Grodzinsky AJ, Burger E, Gaschen V, Hung HH, Hunziker EB (2004) Chondrocyte mechanotransduction: effects of compression on deformation of intracellular organelles and relevance to cellular biosynthesis. Osteoarthr Cartil 12(12):937946

Tan TW, Lai CH, Huang CY, Yang WH, Chen HT, Hsu HC, Fong YC, Tang CH (2009) CTGF enhances migration and MMP-13 upregulation via alphavbeta3 integrin, FAK, ERK, and NFkappaB-dependent pathway in human chondrosarcoma cells. J Cell Biochem 107(2):345-356

Tsang KY, Chan D, Cheslett D, Chan WC, So CL, Melhado IG, Chan TW, Kwan KM, Hunziker EB, Yamada Y, Bateman JF, Cheung KM, Cheah KS (2007) Surviving endoplasmic reticulum stress is coupled to altered chondrocyte differentiation and function. PLoS Biol 5(3): 44

Voncken JW (2011) Genetic modification of the mouse: general technology - pronuclear and blastocyst injection. Methods Mol Biol 693:11-36

Vranka J, Mokashi A, Keene DR, Tufa S, Corson G, Sussman M, Horton WA, Maddox K, Sakai L, Bachinger HP (2001) Selective intracellular retention of extracellular matrix proteins and chaperones associated with pseudoachondroplasia. Matrix Biol 20(7):439-450

Walter P, Ron D (2011) The unfolded protein response: from stress pathway to homeostatic regulation. Science 334(6059):10811086

Wu S, Flint JK, Rezvani G, De Luca F (2007) Nuclear factor-kappaB p65 facilitates longitudinal bone growth by inducing growth plate chondrocyte proliferation and differentiation and by preventing apoptosis. J Biol Chem 282(46):33698-33706

Yang L, Carlson SG, McBurney D, Horton WE Jr (2005) Multiple signals induce endoplasmic reticulum stress in both primary and immortalized chondrocytes resulting in loss of differentiation, impaired cell growth, and apoptosis. J Biol Chem 280(35):31156-31165

Zahir N, Lakins JN, Russell A, Ming W, Chatterjee C, Rozenberg GI, Marinkovich MP, Weaver VM (2003) Autocrine laminin-5 ligates alpha6beta4 integrin and activates RAC and NFkappaB to mediate anchorage-independent survival of mammary tumors. J Cell Biol 163(6):1397-1407 